\title{
Alta prevalencia de infecciones asintomáticas de malaria en la frontera Ecuador Colombia
}

\author{
Bibiana Salazar-Costa[1], Claudia A. Vera-Arias[1], María Isabel Tamayo[1], César A. Yumiseva[1], Jaen Carlos \\ Cagua-Ordoñez[2], Javier Gomez-Obando[3], Fabián E. Sáenz[1] \\ 1. Centro de Investigación para la Salud en América Latina, Pontificia Universidad Católica del Ecuador, \\ Ecuador \\ 2. Dirección de Estrategias de Prevención y Control, Ministerio de Salud Pública, Quito, Ecuador \\ 3. Ministerio de Salud Pública, San Lorenzo, Esmeraldas, Ecuador
}

Doi: https://doi.org/10.23936/pfr.v5i2.157

Recibido: 23/05/2020 Aprobado:29/06/2020

PRÁCTICA FAMILIAR RURAL | Vol.5 | No.2 | Julio 2020 | Recibido: 23/05/2020 | Aprobado:29/06/2020

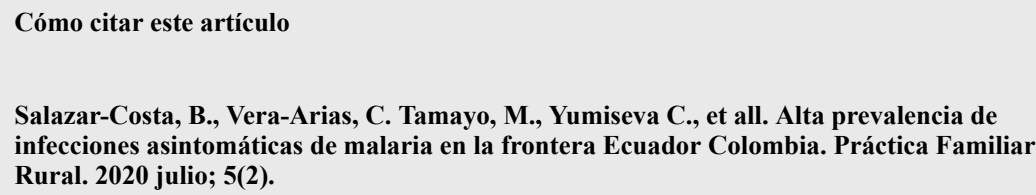

Compartir en:

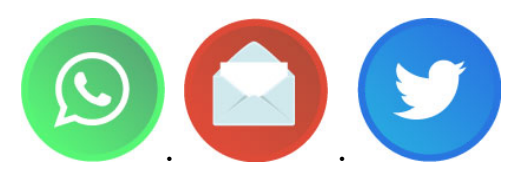

\section{Resumen}

La malaria es una enfermedad que afecta a las zonas tropicales del mundo, siendo un importante problema de salud pública. En los últimos 18 años la incidencia en Ecuador ha disminuido, lo que lo ubicó entre los países con capacidad de eliminar la malaria hasta el 2020. Sin embargo, a partir del 2015 se ha presentado un incremento en el número de casos afectando principalmente a la población más vulnerable de las áreas fronterizas del país. La presencia de infecciones asintomáticas, las infecciones sub microscópicas y asintomáticas constituyen un problema para la eliminación de la enfermedad. En este trabajo se estudió la epidemiología de la malaria en las comunidades del norte de la provincia de Esmeraldas, fronterizas con Colombia, con muestras sanguíneas del 2017 y se aplicó una encuesta epidemiológica de conocimientos, actitudes y prácticas de malaria (CAP) a los jefes de familia. La prevalencia total de malaria en las zonas de estudio obtenida mediante técnicas moleculares fue de $5.83 \%$. Todas las infecciones fueron asintomáticas. La población encuestada presentó un conocimiento básico respecto a la malaria, pero la falta de prácticas para prevenir la malaria es un factor importante que podría dificultar la eliminación de la malaria. Este estudio proporciona información sobre el estado de la malaria en las comunidades de estudio del norte de Esmeraldas que facilitará a la formulación de nuevas estrategias para el control y prevención de la enfermedad. 
Palabras clave: malaria asintomática, Ecuador, Esmeraldas, conocimientos, actitudes y prácticas, prevalencia.

\title{
High prevalence of asymptomatic malaria infections on the Ecuador-Colombia border
}

\begin{abstract}
Malaria is a disease that affects the tropical areas of the world, being a major public health problem. In the last 18 years, the malaria incidence in Ecuador has decreased and Ecuador has been placed among the countries with the capacity to eliminate malaria before 2020. However, since 2015 there has been an increase in the number of malaria cases affecting the most vulnerable population in the border areas of the country. In this work, we studied the epidemiology of malaria in the communities of the north of the province of Esmeraldas, with samples collected in 2017. An epidemiological survey of knowledge, attitudes and practices of malaria (KAP) was applied to household heads. The total prevalence of malaria in the study areas by molecular techniques was $5.83 \%$. All infections were asymptomatic. The surveyed population presented a basic knowledge about malaria but the lack of prevention practices is an important factor that could hinder the elimination of the disease. This study provides information on the state of the disease in the communities of the north of Esmeraldas and will allow the implementation of new strategies of control and prevention of malaria.
\end{abstract}

Keywords: asymptomatic malaria, Ecuador, Esmeraldas, knowledge, attitude and practice, plasmodium, prevalence.

\section{INTRODUCCIÓN}

La incidencia de la malaria en el mundo ha disminuido entre el 2010 y el 2016, en un $18 \%$. Presentando un mayor descenso en Asia sudoriental (48\%), seguido por América (22\%) y África (20\%). A pesar de los logros, a partir del 2014, las regiones de Asia Sudoriental, Pacífico Occidental, África y la región de las Américas reportaron incrementos elevados de casos. La especie $P$. falciparum es más prevalente en Âfrica, mientras que $P$. vivax es predomínate en la región de las Américas (WHO, 2017).

En la región de las Américas, entre los años 2015 y 2017 se reportó un incremento de casos en Colombia, Brasil, Ecuador, México, Nicaragua, Guyana, El Salvador, Haití, Nicaragua, Panamá, Venezuela, Costa Rica y Honduras encontrando, en ciertos países, casos endémicos y en lugares que no se había reportado anteriormente (PAHO, 2018). Países como Venezuela, en el 2017, reportaron más casos que los reportados en los últimos 29 años (PAHO, 2018). La costa pacífica de Colombia y la región amazónica de Perú presentaron mayor transmisión de malaria en el 2017 (PAHO/WHO, 2018). Colombia notificó 55117 casos de malaria (Salas Botero, 2017) y Perú reportó 70700 casos de malaria (Centro Nacional de Epidemiología, 2018).

En Ecuador, la malaria está presente en la Costa y Amazonía, especialmente en cuatro provincias: Morona Santiago, Pastaza, Orellana y Esmeraldas. La especie $P$. vivax está presente con mayor frecuencia en la región Amazónica, mientras que la especie P. falciparum está presente, principalmente, en la provincia de Esmeraldas (OPS/OMS, 2017).

Entre el 2001 y el 2014 Ecuador experimentó una reducción de casos de malaria de más del 99.5\%, y el país fue incluido en la fase de pre-eliminación según la Organización Mundial de la Salud (OMS) en el 2013 (OPS/OMS, 2014). A pesar de la disminución del número de casos a partir del 2015, Ecuador presentó un incremento de casos de malaria. En el 2016, se notificaron 926 casos de malaria, 69\% de estos correspondientes a P. vivax y $31 \%$ a $P$. falciparum. En el 2017, se reportó un incremento de casos de malaria a 1279, el $72 \%$ de los cuales fue ocasionado por $P$. vivax y el $28 \%$ por $P$. falciparum. En el 2018 el número de casos reportados de malaria fue de 1718 (OMS, 2017a; OPS/OMS, 2017, PAHO, 2018) . 
En América Latina se ha reportado presencia de infecciones asintomáticas y sub-microscópicas en Brasil, Perú, Colombia y Venezuela (Recht et al., 2017). En Colombia, las infecciones sub-microscópicas prevalecen en áreas de transmisión baja, siendo importante la vigilancia de estos casos para determinar la incidencia de malaria (Vallejo et al., 2015). La Amazonía peruana presenta una alta prevalencia de asintomáticos en lugares de difícil acceso a un laboratorio (Serra-Casas et al., 2017). En Ecuador reportamos una proporción alta de infecciones asintomáticas en comunidades de El Pedregal, Ricaurte, La Boca y El Guadual en el cantón San Lorenzo (7.4\% de prevalencia), siendo todas infecciones asintomáticas, indicando una posible sub-estimación de casos de malaria en esta zona (Saenz, 2017).

En este estudio realizamos un corte transversal en las poblaciones fronterizas de Mataje y Ricaurte (cantón San Lorenzo) para determinar la prevalencia de Plasmodium spp. en el norte de Esmeraldas mediante PCR anidada. Además determinamos los conocimientos, actitudes, prácticas y características socio-demográficas de la población de estudio.

\section{METODOLOGÍA}

\section{Ética}

Todos los procedimientos realizados en este trabajo de investigación contaron con la aprobación del Comité de Ética de Investigación de la Pontificia Universidad Católica del Ecuador (CEISH-348-2017) y con la aceptación del proyecto por parte del Ministerio de Salud Pública (MSP-DIS-2017-01-0149-O). Los participantes que formaron parte de la investigación, firmaron un consentimiento informado y los menores de edad firmaron un asentimiento.

Area de estudio

El estudio se realizó en las comunidades de Mataje y Ricaurte, ubicadas en el cantón San Lorenzo, provincia de Esmeraldas. Mataje es una población rural, en la frontera con Colombia ubicada junto al río Mataje, con una población de 550 habitantes. El reporte de casos de malaria en Mataje ha sido constante en años recientes: 40 casos reportados en el 2017, 24 en el 2018 y 14 en el 2019. Ricaurte es una comunidad semi urbana en el río Tululbí, ubicada a $15 \mathrm{~km}$ al sur de Mataje, tiene una población de 1300 habitantes. Ricaurte ha reportado casos esporádicos de malaria en años recientes (3 casos reportados en el 2018) (MSP 2019).

Figura 1 . Ubicación geográfica de las comunidades de estudio.

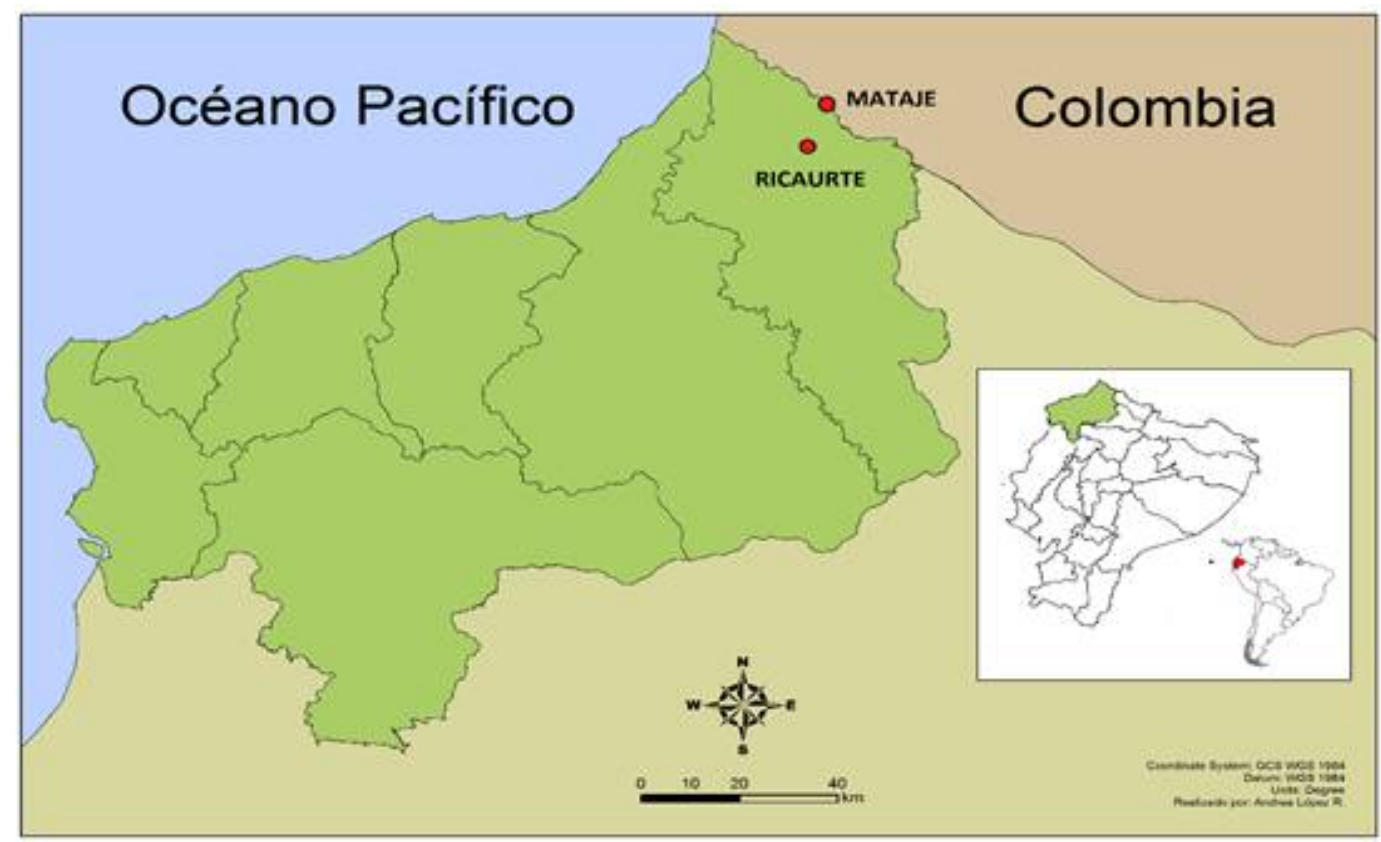

Se tomaron 87 muestras en Mataje y 50 muestras en Ricaurte

\section{Colección de muestras}


Muestras de sangre fueron tomadas aleatoriamente en las comunidades de estudio. El número mínimo de muestras a tomar se definió usando el programa Epidat para obtener una confianza de $95 \%$. Se colectaron 87 muestras en Mataje y 50 en Ricaurte en noviembre de 2017. Se colectó sangre completa en tubo con anticoagulante EDTA por parte del personal del Centro de investigación para la Salud en América Latina (CISeAL) y del Ministerio de Salud Pública (MSP) en habitantes de más de 2 años de edad. Las casas fueron georeferenciadas y localizadas en un mapa.

\section{Encuesta de conocimientos, actitudes y prácticas}

Se realizó una encuesta epidemiológica de conocimientos, actitudes, prácticas y socio-demográfica (CAPs) al jefe de familia o al responsable de cada casa donde se obtuvo la muestra de sangre con la ubicación en GPS para los diferentes análisis en la comunidad de Mataje.

La encuesta constaba de 41 preguntas y estaba dividida en cuatro secciones: conocimientos sobre la transmisión, síntomas, tratamiento y diagnóstico (12 preguntas); actitudes sobre el diagnóstico, prevención y transmisión de la malaria (10 preguntas); prácticas sobre el comportamiento que presentaba la comunidad estudiada frente al tratamiento y prevención de la enfermedad ( 9 preguntas); y características socio-demográficas que nos permitieron observar las características de la población como: servicios básicos, etnicidad y nivel de educación (11 preguntas).

\section{Parasitemia por microscopía}

Se realizó una lectura en el microscopio, en un lente de 100x, de un frotis sanguíneo teñido con Giemsa de gota gruesa, tanto en las placas de las muestras control de P. falciparum como $P$. vivax, preservadas en el laboratorio del CISeAL y previamente observadas por más de una persona, para determinar la presencia del parásito. Posteriormente, se realizó el conteo de los parásitos y leucocitos por campo hasta contar cuantos parásitos estuvieron en 100 leucocitos, y se estimó la densidad parasitaria por micro litro $(\mu \mathrm{L})$ de sangre de las muestras control a través de la fórmula descrita en Alger (2001):

$$
\left(N^{\circ} \text { de parásitos } * 8000 \text { leucocitos } / \mu L \text { de sangre) } / N^{\circ}\right. \text { de leucocitos }
$$

\section{Parasitemia mediante métodos moleculares}

Se realizó la extracción de ADN a partir de sangre mediante el Pure Link® Genomic DNA Mini Kit (Invitrogen), siguiendo el protocolo del fabricante.

\section{PCR anidada para determinar la presencia de Plasmodium spp.}

Se realizó una PCR anidada mediante la técnica de Snounou (1996) con modificaciones según Ruybal-Pesántez y colaboradores (2017):

Los parámetros que se utilizaron en el perfil térmico para las dos PCR fueron: $95{ }^{\circ} \mathrm{C}$ por 2 minutos de denaturación inicial, 25 ciclos de: $58^{\circ} \mathrm{C}$ por 2 minutos, $72^{\circ} \mathrm{C}$ por 5 minutos $94^{\circ} \mathrm{C}$ por 1 minuto; $58^{\circ} \mathrm{C}$ por 2 minutos, $72^{\circ} \mathrm{C}$ por 2 minutos y $4^{\circ} \mathrm{C}$.

Los resultados se observaron mediante una amplificación de $\sim 100$ pb para P. vivax y 200 pb para P. falciparum en el gel de electroforesis.

\section{Análisis de datos}

Los datos obtenidos en las encuestas fueron ingresados en el sistema OpenClinica administrado por el CISeAL. Para el análisis, los datos después de su ingreso se importaron al software Microsoft Excel y en los análisis estadísticos se utilizó el programa SPSS Statistical Software 22. El test estadístico utilizado en el estudio fue la prueba de Ji cuadrado.

\section{RESULTADOS}

\section{Población de estudio}


Se obtuvieron 137 muestras sanguíneas pertenecientes a las comunidades de Mataje $(n=87)$ y Ricaurte $(n=50)$, ubicadas en la zona fronteriza del cantón San Lorenzo, provincia de Esmeraldas, región costanera del Ecuador (figura 1). El promedio de edad de los voluntarios fue de 26 años; y la mayoría de muestras de sangre fueron de niños entre 6 a 10 años de edad, siendo el género femenino el más representado (56\%) (figura 2). Todos los individuos encuestados se consideraron de raza negra o mulata. El 50\% de jefes de familia encuestados se denominaron como propietarios de minas. El grado máximo de escolaridad de las poblaciones fue de secundaria completa o incompleta (50\%). Todas las viviendas encuestadas tenían electricidad y el $75 \%$ eran de ladrillo o cemento. El 94\% de las viviendas contaban con un acueducto como servicio público pero ninguna contaba con alcantarillado.

Figura 2. Frecuencia de edades de los participantes

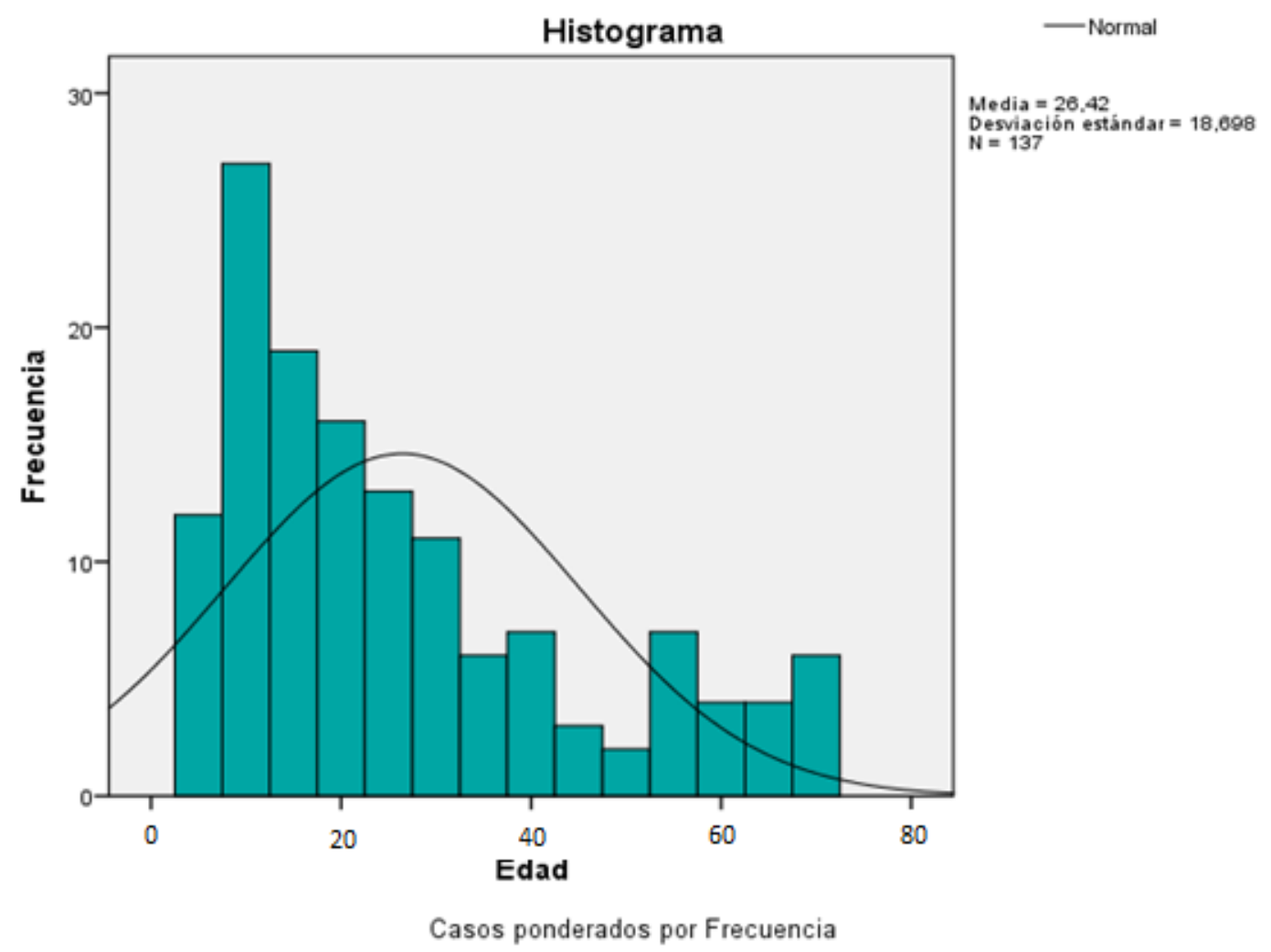

La mayoría de muestras fueron tomadas en personas entre 10 y 30 años. La media poblacional fue 26 años.

\section{Prevalencia de Plasmodium spp. en las comunidades de estudio}

No se detectaron infecciones por microscopía. Todas las láminas examinadas fueron negativas.

El estudio de prevalencia detectó 8 personas (5.83\%) infectadas con Plasmodium spp.mediante PCR anidada. Dos infecciones (1.45\%) fueron causadas por $P$. vivax y 6 infecciones (4.38\%) por P. falciparum (figura 3 ).

En la comunidad de Mataje se obtuvo una prevalencia de $5.75 \%$. En esta comunidad se encontraron dos infecciones por $P$. vivax $(2.30 \%)$ y 3 infecciones por $P$. falciparum (3.45\%), mientras que en la comunidad de Ricaurte se encontraron únicamente infecciones causadas por $P$. falciparum (6\%). Todas las infecciones positivas encontradas fueron de pacientes asintomáticos.

Figura 3. Prevalencia de $P$. vivax y $P$. falciparum en las comunidades de Mataje y Ricaurte 


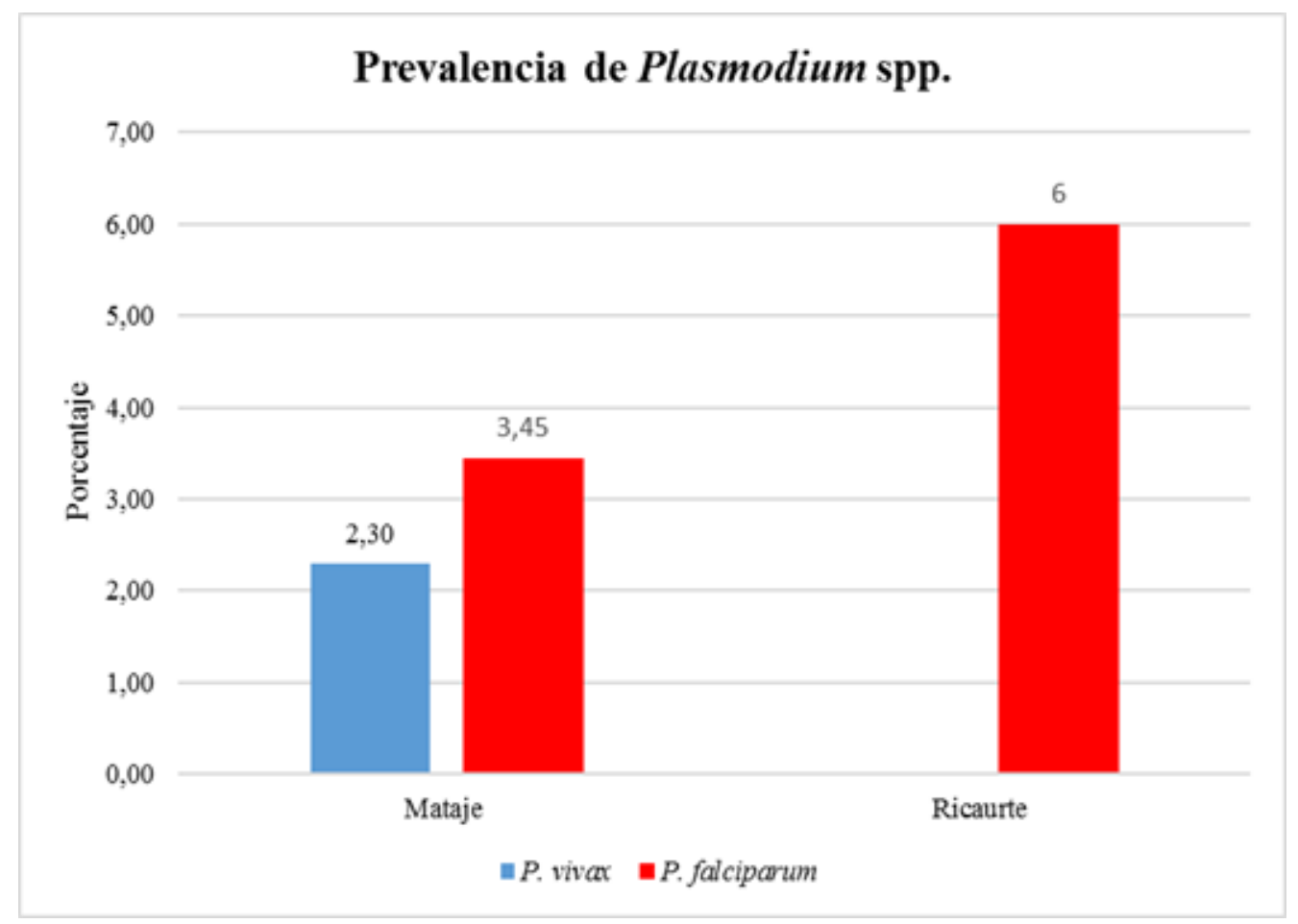

P. falciparum fue la especie más prevalente en ambas comunidades (PCR anidada).

\section{Localización geográfica de las infecciones}

Las casas con infecciones en la comunidad de Mataje, se encontraron relativamente cercanas entre sí y al cuerpo de agua más cercano (río Mataje). La comunidad de Mataje presentó dos zonas principales donde se ubicaron las casas positivas: un de las casas positiva para $P$. falciparum se encontró al noroeste de la comunidad, cerca de la frontera con Colombia y esta se encontró a una distancia de $583 \mathrm{~m}$ del resto de las casas que presentaron casos positivos (figura 4). Mientras que al noreste de la comunidad se encontraron casas positivas para P. falciparum y $P$. vivax que presentaron una distancia de $133 \mathrm{~m}$ entre sí (figura 5).

En la comunidad de Ricaurte, la vivienda donde se encontraron los casos positivos estaba alejada de las otras que pertenecieron al estudio. Esta vivienda estaba a una distancia de $185 \mathrm{~m}$ del cuerpo de agua más cercano (río Tululbí) (figura 5). Se observó que en cada casa con casos positivos, presentaron uno o dos casos de malaria.

Figura 4. Ubicación geográfica de las casas encuestadas de Mataje 


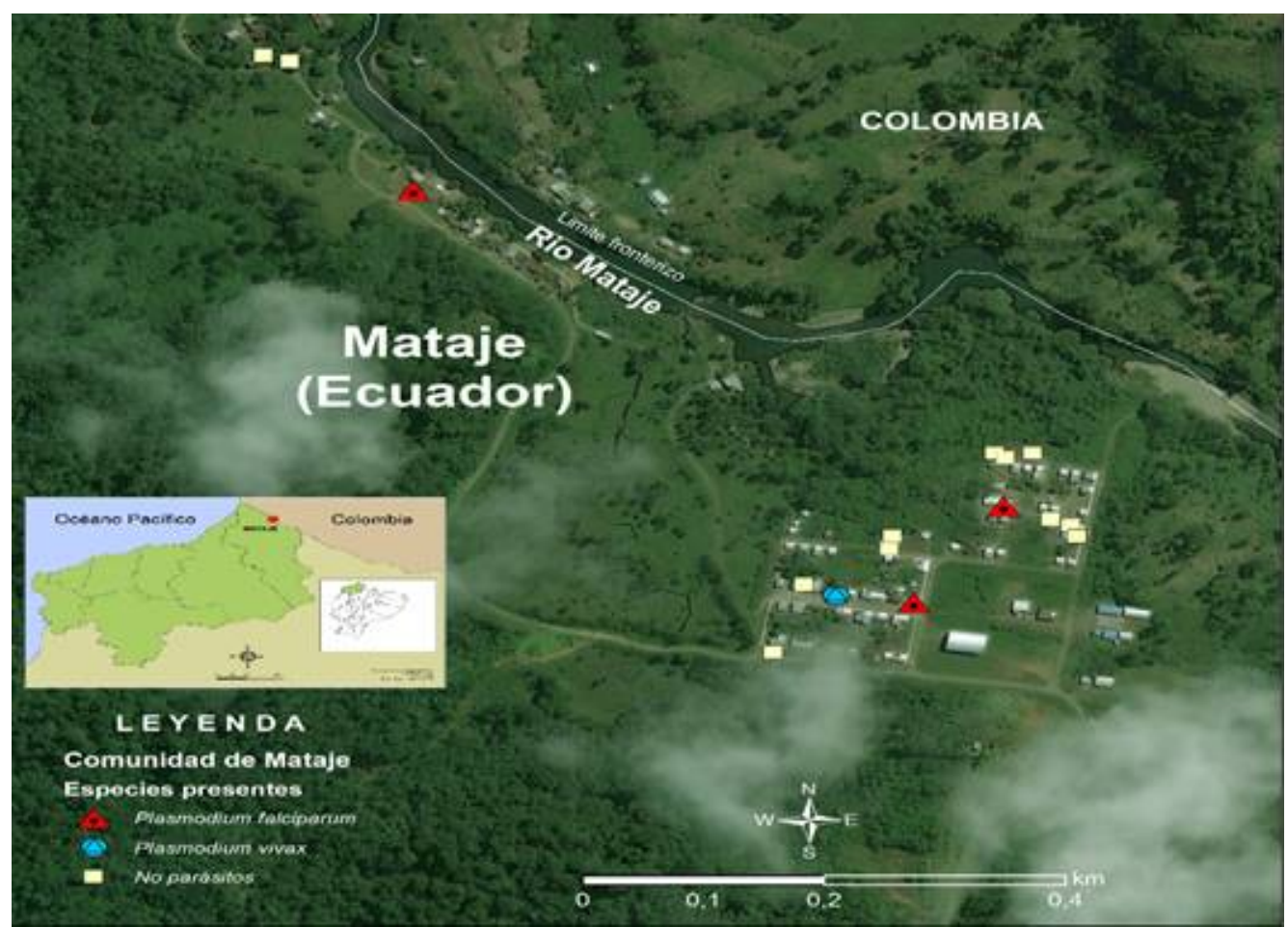

Localización de los casos positivos de P. falciparum y P. vivax.

Figura 5. Ubicación geográfica de las casas positivas para malaria de Ricaurte

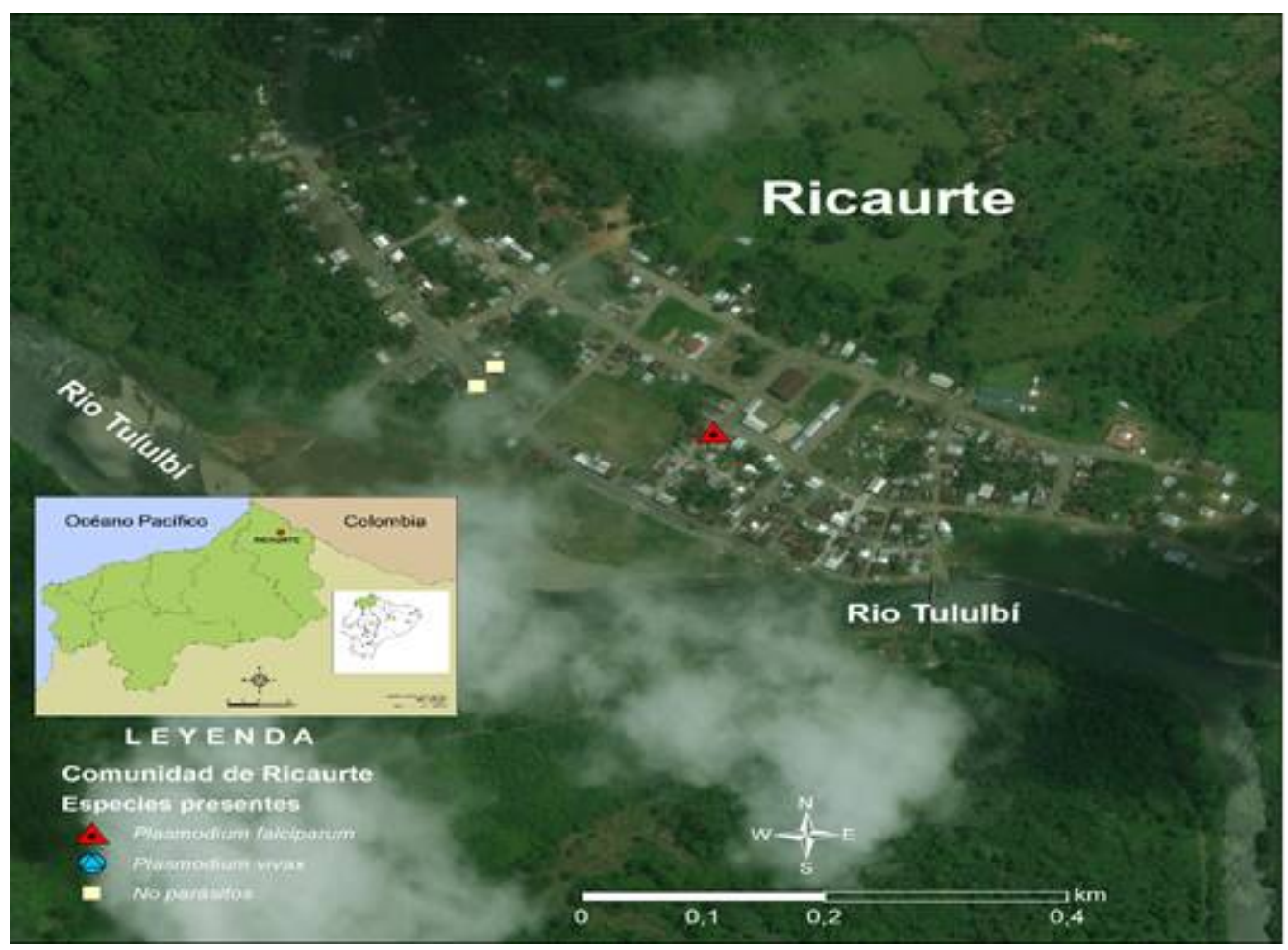

Encuestas de conocimientos, actitudes y prácticas

La mayor cantidad de encuestados respondieron correctamente con respecto a los conocimientos de malaria. La amplia mayoría de los pobladores (60-100\%) conocen el modo de transmisión de la enfermedad, los síntomas, el tratamiento y las acciones de prevención y diagnóstico que deben ser tomadas (Tabla 1).

Tabla 1. Respuestas de la mayoría de encuestados acerca del conocimiento de malaria en la comunidad de Mataje. 


\begin{tabular}{|c|c|c|}
\hline Pregunta & Respuesta & Porcentaje \\
\hline Transmisión de malaria & Picadura de mosquito & 87,5 \\
\hline Síntomas & Dolor de cabeza & 62,5 \\
\hline Acción en caso de síntomas & $\begin{array}{l}\text { Ir al microscopista u otro } \\
\text { funcionario de salud }\end{array}$ & 93,75 \\
\hline Presencia de microscopista en la comunidad & $\mathrm{Si}$ & 93,75 \\
\hline Costo de examen de malaria & No & 100 \\
\hline ¿Con qué se cura la malaria? & Con pastillas & 68,75 \\
\hline Qué ocurre si no se trata la malaria & Se agrava y puede morir & 93,75 \\
\hline $\begin{array}{l}\text { Qué se puede hacer dentro de la vivienda para } \\
\text { evitar la malaria? }\end{array}$ & ${ }^{\mathrm{a}}$ Usar toldillo o mosquitero & 43,75 \\
\hline $\begin{array}{l}\text { Para usted qué se puede hacer fuera de la } \\
\text { vivienda para prevenir la malaria? }\end{array}$ & $\begin{array}{l}\text { aEvitar aguas estancadas, } \\
\text { secar charcos }\end{array}$ & 81,25 \\
\hline $\begin{array}{l}\text { ¿Quiénes son los responsables de controlar la } \\
\text { malaria? }\end{array}$ & ${ }^{a}$ Centro de salud & 68,75 \\
\hline ¿Quién le enseñó lo que sabe de malaria? & Trabajadores de la Salud & 87,5 \\
\hline La malaria puede dar más de una vez. & $\mathrm{Si}$ & 68,75 \\
\hline $\begin{array}{l}\text { La malaria se transmite a través de contacto } \\
\text { físico. }\end{array}$ & ${ }^{\mathrm{o}} \mathrm{No}$ & 75 \\
\hline Los medicamentos curan la malaria. & $\mathrm{Si}$ & 87,5 \\
\hline
\end{tabular}

De acuerdo a la encuesta de actitudes y prácticas de la población con respecto a la malaria se puede ver la mayoría de la población encuestada tiene actitudes y prácticas acertadas con respecto a la prevención de la enfermedad. Entre las respuestas destaca que $56 \%$ no consideran a la malaria como algo normal, $81 \%$ usan toldo y la mayoría realizan acciones para prevenir la enfermedad fuera de la vivienda. Sin embargo, un porcentaje declaran haber obtenido medicinas sin prescripción médica y $44 \%$ señala no haber acudido a personal de salud cuando presentaron síntomas de la enfermedad (tabla 2).

Tabla 2. Respuestas de la mayoría de encuestados acerca de las actitudes y prácticas de malaria en la comunidad de Mataje.

\begin{tabular}{llc}
\multicolumn{1}{c}{ Pregunta } & \multicolumn{1}{c}{ Respuesta } & Porcentaje \\
Enfermarse de malaria o paludismo es algo común y normal. & $\mathrm{No}$ & 56,25 \\
Charcas o agua acumulada facilita la transmisión & $\mathrm{Si}$ & 93,75 \\
Usar toldillo es molesto. & $\mathrm{No}$ & 62,5 \\
La presencia de los mosquitos o zancudos le molesta. & $\mathrm{Si}$ & 93,75 \\
La Gota Gruesa es necesaria para diagnosticar paludismo & $\mathrm{Si}$ & 81,25 \\
Se debe terminar los medicamentos & $\mathrm{Si}$ & 93,75 \\
Ha comprado pastillas sin prescripción médica & $\mathrm{No}$ & 75,00 \\
Dentro de la vivienda & Usa toldo & 81,25 \\
Fuera de la vivienda & Seca aguas & 62,5 \\
& estancadas & \\
A quien acudió primero si estuvo enfermo de malaria? & Personal de & 81,25 \\
La última vez que tuvo malaria se hizo tomar gota gruesa. & salud & No \\
Medio para desplazarse al diagnóstico & A pie & 43,75 \\
Tiempo al sitio de diagnóstico & Menos de una & 93,75 \\
Por qué ha suspendido el tratamiento & hora & 100,00 \\
Ha recibido gratis el tratamiento del personal de salud & Se siente mejor & 62,5 \\
& Si & 93,75
\end{tabular}

\section{DISCUSIÓN}

En este estudio determinamos la prevalencia de infecciones de malaria y los conocimientos, actitudes y prácticas con respecto a esta enfermedad en poblaciones fronterizas de la provincia de Esmeraldas. 
Las infecciones asintomáticas son difíciles de detectar debido a que presentan bajas parasitemias y son consideradas como reservorios que permiten la transmisión de la enfermedad (Laishram et al., 2012; Sáenz et al., 2017). Estas infecciones pueden ser detectadas por técnicas moleculares. La PCR, empleada para la observación de presencia o ausencia de los parásitos, utilizando secuencias específicas que permiten la determinación precisa de la especie y a su vez permite la cuantificación de baja carga parasitaria hasta 1 parásito / $\mu \mathrm{L}$ de sangre en infecciones asintomáticas y submicroscópicas, las cuales son difíciles de detectar a causa de sus bajas parasitemias (Baird et al., 2016; Vásquez-Jiménez et al., 2016). El uso de la PCR permite la detección y amplificación del ADN del parásito dentro de una muestra, teniendo mayor especificidad y sensibilidad que otras pruebas como la microscopía y pruebas rápidas (Baird et al., 2016; Moreira, Abo-Shehada, Price, \& Drakeley, 2015)

Este estudio confirma la presencia de infecciones asintomáticas y submicroscópicas en dos comunidades del norte de la provincia de Esmeraldas, con una prevalencia de 5.8\%. En un estudio previo, en poblaciones cercanas a las de este estudio se detectó un prevalencia de 7.2\% en cuatro comunidades del cantón San Lorenzo (Sáenz et al., 2017). Los resultados de este estudio son comparables a los reportados anteriormente en la misma zona. Sin embargo, este es el primer reporte de prevalencia molecular de malaria en la comunidad de Mataje. Cabe recalcar que al igual que en el estudio de Saenz et al.,2017, todas pertenecían a pacientes asintomáticos A diferencia de estudios anteriores realizados en la misma área, en este estudio se reportó una mayor prevalencia de $P$. falciparum que de $P$. vivax. Es importante notar que se encontraron dos infecciones por $P$. vivax, a pesar de que infecciones por $P$. vivax no son comúnmente reportadas en esta área.

En países aledaños como Colombia, en regiones de Tumaco, Tierraalta y Buenaventura, se reportó una prevalencia de infecciones sub-microscópicas con Plasmodium spp. de 9.7\%, donde la tasa de positividad varió entre 3\% y 20\% al utilizar PCR en tiempo real (Vallejo et al., 2015). En la Amazonía de Perú, en comunidades de Cahuide y Lupuna del departamento de Loreto, se encontró una prevalencia de $10.4 \%$ de infecciones asintomáticas y submicroscópicas con Plasmodium spp. (Rovira-Vallbona et al., 2017).

Las casas que presentaron infecciones tanto en Mataje como Ricaurte estaban cercanas al río Mataje y al río Tululbí respectivamente, indicando mayor probabilidad de que existan cuerpos de agua cercanos a sus hogares donde los mosquitos depositen sus huevos y puedan ser lugares para dispersión de los insectos.

En lugares endémicos la población adquiere inmunidad parcial a la exposición a la enfermedad, siendo posible la presencia de malaria asintomática. La presencia de infecciones asintomáticas podría indicar que la población ha desarrollado inmunidad ante la enfermedad, a pesar de su baja intensidad en la transmisión. Sáenz y colaboradores (2017), en el Ecuador, demostraron que más de un tercio de la población de estudio mostró títulos positivos de anticuerpos contra las especies $P$. falciparum y $P$. vivax, confirmando el desarrollo de la inmunidad de la población ante la malaria. Las infecciones sin síntomas representan un problema en la eliminación de la enfermedad, debido a que la población asintomática puede actuar como un reservorio en la transmisión de la enfermedad (Lindblade, Steinhardt, Samuels, Kachur, \& Slutsker, 2013).

Un factor de riesgo para la transmisión de la malaria en la zona es el trabajo en minas. En este caso la mitad de la población encuestada indicó que era dueños de minas, confirmando una posible alta exposición en la zona debido a esta actividad (Dawaki et al., 2016). La presencia del parásito en personas que trabajan en las minas es un factor limitante en la eliminación de la malaria por la alta movilidad y difícil acceso al lugar.

El nivel de escolaridad se podría considerar como un factor de riesgo asociado a la prevalencia de la enfermedad en la zona ya que permite observar el conocimiento que presenta la comunidad para resolver un problema o tomar decisiones que beneficien ante una dificultad (Poveda, 2015). Comunidades con bajos recursos económicos que poseen un nivel menor de escolaridad son más susceptibles a ciertas enfermedades como la malaria. La mayoría de la población de Mataje alcanzó un nivel de escolaridad de primaria y parte de la secundaria.

Debido a la exposición histórica a la malaria en la zona, la mayoría de la población de estudio presentó un conocimiento adecuado de la enfermedad tanto en la compresión de la transmisión, síntomas, tratamiento y prevención de la misma. el conocimiento sobre la enfermedad es esencial para la práctica de medidas preventivas y la búsqueda pertinente de ayuda por parte del personal de salud (Soleimani-Ahmadi, Vatandoost, Zare, Alizadeh, \& Salehi, 2014). 
La gran mayoría de la población encuestada declaró tener prácticas y actitudes con respecto a la prevención, diagnóstico y tratamiento de la malaria en la zona. Sin embargo, un porcentaje menor $(25 \%)$ adquirió fármacos sin receta médica para tratar la enfermedad. Este número es muy inferior al reportado en países de Africa (Adedotun, Morenikeji, \& Odaibo, 2010), pero puede representar un factor de riesgo debido a que se los parásitos podrían generar resistencia a las diferentes medicinas (Dawaki et al., 2016). El 43,75\% de la población encuestada no acudió al personal de salud cuando tuvo síntomas de malaria y no se realizó ningún examen. El examen sanguíneo de la gota gruesa es esencial para diagnosticar la enfermedad en la zona, al no realizarse esta prueba de diagnóstico la enfermedad puede complicarse y la población sana se compromete al correr el riesgo de contagiarse, por ende, aumentar los casos. La comunidad de Mataje presenta accesibilidad adecuada al diagnóstico y tratamiento, lo que facilita en control de la enfermedad en la zona.

El presente estudio proporciona una mejor comprensión de la epidemiología de la malaria en las poblaciones fronterizas de Mataje y Ricaurte, en el cantón San Lorenzo, zona de tránsito legal e ilegal entre Ecuador y Colombia. Detectamos un porcentaje considerable de infecciones asintomáticas, lo cual confirma la transmisión continua de la enfermedad. La presencia de infecciones asintomáticas representa un reto para la eliminación e la malaria en la zona ya que puede mantener la transmisión de la malaria en las zonas fronterizas. Además, la educación continua para el conocimiento y la prevención de la malaria es necesaria para aportar a la lucha contra la enfermedad. Un trabajo conjunto entre países vecinos será la mejor estrategia para alcanzar la eliminación de la malaria en las zonas fronterizas.

\section{REFERENCIAS:}

Adedotun, A. A., Morenikeji, O. A., \& Odaibo, A. B. (2010). Knowledge, attitudes and practices about malaria in an urban community in saouth-western Nigeria. Journal of Vector Borne Diseases, 47(3), 155.

Alger, J. (2001). Densidad Parasitaria en Malaria : Métodos de determinación y su interpretación, 118-120.

Baird, J. K., Valecha, N., Duparc, S., White, N. J., \& Price, R. N. (2016). Diagnosis and Treatment of Plasmodium vivax Malaria. The American Journal of Tropical Medicine and Hygiene, 95(6 Suppl), 35-51. https://doi.org/10.4269/ajtmh.16-0171

Centro Nacional de Epidemiología, P. y C. de E. (2018). Número de casos por tipo de malaria, (48887).

Dawaki, S., Al-Mekhlafi, H. M., Ithoi, I., Ibrahim, J., Atroosh, W. M., Abdulsalam, A. M., ... Lau, Y.-L. (2016). Is Nigeria winning the battle against malaria? Prevalence, risk factors and KAP assessment among Hausa communities in Kano State. Malaria Journal, 15(1), 351. https://doi.org/10.1186/s12936-016-1394-3

Laishram, D. D., Sutton, P. L., Nanda, N., Sharma, V. L., Sobti, R. C., Carlton, J. M., \& Joshi, H. (2012). The complexities of malaria disease manifestations with a focus on asymptomatic malaria. Malaria Journal, 11(1), 29. https://doi.org/10.1186/1475-2875-11-29

Lindblade, K. A., Steinhardt, L., Samuels, A., Kachur, S. P., \& Slutsker, L. (2013). The silent threat: asymptomatic parasitemia and malaria transmission. Expert Review of Anti-infective Therapy, 11(6), 623-639. https://doi.org/10.1586/eri.13.45

Ministerio de Salud Pública del Ecuador (2019). SIVE-ALERTA. Gaceta epidemiológica de vectores. Recuperado a partir de: https://www.salud.gob.ec/wp- content/uploads/2019/01/GACETA-VECTORES-SE52.pdf

Moreira, C. M., Abo-Shehada, M., Price, R. N., \& Drakeley, C. J. (2015). A systematic review of submicroscopic Plasmodium vivax infection. Malaria journal, 14, 360. https://doi.org/10.1186/s12936-015-0884-z

OMS. (2017a). Control y eliminación del paludismo por Plasmodium vivax: informe técnico ["OMS | Control y eliminación del paludismo por Plasmodium vivax: informe técnico". WHO. http://www.who.int/malaria/publications/atoz/9789241509244/es/.].

OPS/OMS. (2014). Informe de la situación de la Malaria en las Américas. Recuperado a partir de http://iris.paho.org/xmlui/handle/123456789/33881

OPS/OMS. (2017a). Alerta Epidemiológica Aumento de casos de malaria. Recuperado a partir de http://www.paho.org/hq/index.php?option=com_docman\&task=doc_view\&Itemid=270\&gid=38148\&lang=es PAHO. (2018). Actualización Epidemiológica. Aumento de malaria en las Américas, 5. Recuperado a partir de https://www.paho.org/hq/index.php?

option=com_docman\&task=doc_view\&Itemid=270\&gid=43437\&lang=es\%0Ahttp://www.paho.org/hq/index.php? option $=$ com_docman\&task $=$ doc_view\&Itemid $=270 \&$ gid $=43437 \&$ lang $=$ es

Poveda-Loayza, A. C. (2015). Determinacion de la prevalencia de Plasmodium spp mediante microscopia y su relacion con conocimientos, actitudes y prácticas de malaria como posibles factores de riesgo en cuatro comunidades de San Lorenzo provincia de Esmeraldas. ESPE.

Recht, J., Siqueira, A. M., Monteiro, W. M., Herrera, S. M., Herrera, S., \& Lacerda, M. V. G. (2017). Malaria in 
Brazil, Colombia, Peru and Venezuela: Current challenges in malaria control and elimination. Malaria Journal, 16(1), 1-18. https://doi.org/10.1186/s12936-017-1925-6

Rovira-Vallbona, E., Contreras-Mancilla, J. J., Ramirez, R., Guzmán-Guzmán, M., Carrasco-Escobar, G., LlanosCuentas, A., ... Rosanas-Urgell, A. (2017). Predominance of asymptomatic and sub-microscopic infections characterizes the Plasmodium gametocyte reservoir in the Peruvian Amazon. PLOS Neglected Tropical Diseases, 11(7), e0005674. https://doi.org/10.1371/journal.pntd.0005674

Ruybal-Pesántez, S., Tiedje, K. E., Rorick, M. M., Amenga-Etego, L., Ghansah, A., Oduro, A. R., ... Day, K. P. (2017). Lack of geospatial population structure yet significant linkage disequilibrium in the reservoir of plasmodium falciparum in Bongo District, Ghana. American Journal of Tropical Medicine and Hygiene, 97(4), 1180-1189. https://doi.org/10.4269/ajtmh.17-0119

Sáenz, F. E., Arévalo-Cortés, A., Valenzuela, G., Vallejo, A. F., Castellanos, A., Poveda-Loayza, A. C., ... Herrera, S. (2017). Malaria epidemiology in low-endemicity areas of the northern coast of Ecuador: high prevalence of asymptomatic infections. Malaria Journal, 16(1), 300. https://doi.org/10.1186/s12936-017-1947-0

Salas Botero, D. (2017). Informe De Evento Malaria, Colombia, 2017. Recuperado a partir de https://www.ins.gov.co/buscador-eventos/Informesdeevento/MALARIA 2017.pdf

Serra-Casas, E., Manrique, P., Ding, X. C., Carrasco-Escobar, G., Alava, F., Gave, A., ... Gamboa, D. (2017). Loop-mediated isothermal DNA amplification for asymptomatic malaria detection in challenging field settings: Technical performance and pilot implementation in the Peruvian Amazon. PLOS ONE, 12(10), e0185742. https://doi.org/10.1371/journal.pone.0185742

Snounou G. Detection and identification of the four malaria parasite species infecting humans by PCR amplification. Methods Mol Biol. 1996;50:263-91.

Soleimani-Ahmadi, M., Vatandoost, H., Zare, M., Alizadeh, A., \& Salehi, M. (2014). Community knowledge and practices regarding malaria and long-lasting insecticidal nets during malaria elimination programme in an endemic area in Iran. Malaria Journal, 13(1), 511. https://doi.org/10.1186/1475-2875-13-511

Vallejo, Chaparro, P. E., Benavides, Y., Álvarez, Á., Quintero, J., Padilla, J., ... Herrera, S. (2015). High prevalence of sub-microscopic infections in Colombia. Malaria Journal, 14(1), 201. https://doi.org/10.1186/s12936-015-0711-6

Vásquez-Jiménez, J. M., Arévalo-Herrera, M., Henao-Giraldo, J., Molina-Gómez, K., Arce-Plata, M., Vallejo, A. F., \& Herrera, S. (2016). Consistent prevalence of asymptomatic infections in malaria endemic populations in Colombia over time. Malaria Journal, 15(1), 70. https://doi.org/10.1186/s12936-016-1124-x

WHO. (2017). World Malaria Report 2017. World Health Organization. https://doi.org/10.1071/EC12504

WHO. (2018). OMS | Preparados para vencer al paludismo: Día Mundial del Paludismo 2018. WHO. Recuperado a partir de http://www.who.int/malaria/media/world-malaria-day-2018/es/

\section{AGRADECIMIENTOS:}

Agradecemos al personal del Distrito de Salud 08D05, San Lorenzo, Ministerio de Salud Pública que participó en la toma de muestras. Agradecemos a todos los voluntarios que participaron en el estudio.

FINANCIAMIENTO:

Este estudio fue financiado por la Pontificia Universidad Católica del Ecuador, proyectos M13416 y N13416

\section{CONTRIBUCIONES DE LOS AUTORES}

FES, CAV, JCC y JG diseñaron el estudio. BES y FES escribieron el manuscrito. BES, CAV, MIT y JG realizaron el trabajo de campo. BES y FES analizaron los datos. 